OPEN ACCESS

Vol. 5 No. 1, April 2017

Hal. 1 - 16
JAFFA

Journal of Auditing, Finance, and Forensic Accounting

E-ISSN: 2461-0607 ISSN: 2339-2886

http://jaffa.trunojoyo.ac.id/jaffa

\title{
BANKRUPTCY ANALYSIS USING ALTMAN Z SCORE MODEL IN MANUFACTURING COMPANY AT INDONESIA STOCK EXCHANGE IN 2013-2014
}

\section{Suci Kurniawati}

STIE Indonesia Rawamangun

\section{Article Info:}

Received: 7 October 2016

in revised form: 17 December 2016

Accepted: 22 February 2017

Available Online: 12 November 2017

\section{Keywords:}

financial distress, $z$-score, manufacturing

\section{Corresponding Author:}

Email: suci_kurniawati@yahoo.co.id
Abstract; Analysis of financial distress is very important, because it enables to assess an indication of the company's financial distress, how the indication of financial distress using Altman $z$-score in industry manufacturing sector in 20132014, and whether the Altman $z$-score model can be used as a tool in predicting the tendency of financial distress. The purpose of this study is to analyze the financial distress of 125 manufacturing companies with different sectors and subsectors using Altman Z-Score model in 2013 and 2014. The source of data used was secondary data, such as financial statements of manufacturing companies' publication issued by BEI and obtained from the internet by downloading through the website: www.idx.com. This study employed descriptive quantitative method. The findings of the $Z$-Score index on manufacturing companies in 2013 were occupied by PT. Intan Wijaya International Tbk. in chemical subsector, and in 2014 Herbal and Pharmaceutical Industry of PT Sido Muncul Tbk. was the first highest rank and healthy condition. Whereas the lowest rank was PT. Asia Pacific Fiber Tbk. in textile and garment sub-sector in 2013 and 2014, having financial distress condition. The findings of this study are not consistent or even in accordance with the reality which shows that the Altman method cannot be used as a tool to indicate a tendency towards company's financial distress.

Abstrak; Analisis kebangkrutan sangat penting, karena dapat menilai indikasi kesulitan keuangan perusahaan, bagaimana indikasi kesulitan keuangan menggunakan Altman $z$ - score di industri sektor manufaktur pada tahun 2013-2014, dan apakah model Altman $z$ - score dapat digunakan sebagai alat dalam memprediksi kecenderungan kesulitan keuangan. Tujuan dari penelitian ini adalah untuk menganalisis kebangkrutan perusahaan manufaktur sebanyak 125 perusahaan dengan sektor dan subsektor yang berbeda dengan menggunakan model ALTMAN Z-Score pada tahun 2013 dan 2014. Sumber data yang digunakan adalah data sekunder yakni berupa data Laporan Keuangan Publikasi Perusahaan Manufaktur yang diterbitkan Bursa Efek Indonesia dan diperoleh dari media internet dengan mendownload melalui website: www.idx.com. Penelitian ini menggunakan metode deskriptif kuantitatif. Hasil index ZScore pada perusahaan manufaktur tahun 2013 diduduki oleh PT. Intan Wijaya Internasional Tbk pada subsector kimia dan PT. Industri Jamu \& Farmasi Sido Muncul Tbk pada subsektor farmasi tahun 2014 dengan peringkat pertama tertinggi dengan kondisi sehat, sedangkan yang menduduki peringkat paling terakhir dan terendah di perusahaan manufaktur BEI adalah PT. Asia Pacific Fiber Tbk pada subsektor tekstil \& garmen pada tahun 2013 dan 2014 dengan kondisi bangkrut. Serta hasil penelitian ini tidak konsisten/ sesuai dengan kenyataan sebenarnya yang menunjukkan bahwa metode altman tidak dapat dijadikan alat untuk mengindikasikan kecendrungan terhadap kebangkrutan. 


\section{INTRODUCTION}

Company's bankruptcy is a phenomenon that commonly occurs in the business's world that is influenced by internal or external factor. For example, there is an increase in raw material cost, wage cost, or other costs without being offset by company's ability, or competitor's product's existence that is superior which consequently affects the sales and manager's incapability to manage a company. This particular problem will directly affect company's performance reduction which leads into bankruptcy.

To anticipate the possibility of bankruptcy a company must have early preparation to prevent unexpected things happen. A company is expected to have an access on company's ongoing situation to obtain a better picture from company's current situation, thus it will lead to a right decision to keep a company stay alive and stay compete in business.

One of bankruptcy models proven to give many benefits is the Z-Score model. This model is developed by Edward I Altman, a financial economist. This model is a discriminant multiple statistical techniques that combine several variables effect. It is a financial analysis which has been widely used in the USA. In the study, Altman took a sample of 66 companies which half of it has gone bankrupt. Based on Multiple Discriminant Analysis model, fifth financial ratio's coefficient is determined later. The addition and multiplication between the coefficient and financial ratio create multivariate score. Altman, mentioned this multivariate score as $Z$-Score.

Research on Altman Z-Score method in Indonesia, is conducted by Usman (2013). The result shows that in 2013 as many as $54.5 \%$ company are healthy, $9.1 \%$ is gray area and $36.4 \%$ is in bankrupt. PT Malindo Feedmill Tbk, PT Gudang Garam Tbk, PT Hanjaya Mandala Sampoerna Tbk, PT Multi Bintang Indonesia Tbk, and PT Delta Djakarta Tbk are predicted as healthy company. Further research by Silaban (2014) on bankruptcy analysis uses Altman Z-Score Model, a case study in Perusahaan Telekomunikasi. The analysis shows that between 2010-2012 company's health is not in a good position. If a company is in gray area in 2010, it means that it decreases in the next year, therefore, the company will be bankrupt in 2012. Telkom is in healthy zone and increasing every year, meanwhile, Indosat is in unhealthy zone by z-score that always increases every year. Next study by Jaya (2014) on financial report as bankruptcy prediction uses Altman model. The result of the above study indicates that Altman model can be used as a tool to predict company's bankruptcy.

Some studies use sampling from manufacture industry because the growth rate only reaches $6,2 \%$ in 2013 . Various problems occur from laborer to energy that inhibits manufacturer's growth. One of the problems is provincial minimum wage increases in some areas that reach $40 \%$ to make industrial expense increases, especially for a laborintensive industry that use a lot of manpower to operate. Other problem such as electricity tariff increases along with the rise in gas price increases. With this uncertain situation and condition experienced by manufacturing industry, it becomes heavy to grow thus the industry must adapt to the pressure. Meanwhile, sluggish infrastructure developments do not only make the industry's growth trimmed but also make the high cost of economy and logistic.(http://www.kemenperin.go.id/)

The bankruptcy analysis is very important because it indicates the company's bankruptcy, whether it is threatened by bankruptcy or not. When a company's file for bankruptcy, there are lots of harmed parties such as manager, investor, creditor, and even the employees. Based on the problems above, the researcher considers conducting a study on how is bankruptcy's indication uses Altman Z-Score model in manufacture industry in 2013 and 2014; whether Altman Z-Score Model can be used as a tool to predict company's bankruptcy trend. This study is conducted by analyzing bankruptcy using Altman Z-Score Model in manufacture industry listed at Indonesia Stock Exchange in 2013-2014.

The study's research problem is: Is the Altman Z-Score model can be used as a tool to predict company's bankruptcy trend? While the objective of the study is whether Altman Z-Score Model can be used as a tool in predicting company's bankruptcy trend. 


\section{RESEARCH METHOD}

\section{Research Population}

The population used in this study was the company engaged in sector that listed at Indonesia Stock Exchange (BEI) in the 2013-2014 period as many as 139 companies.

\section{Research Sampling}

Sampling technique used in the study was purposive sampling, that was a carefully selected sample relevant to research design and was expected to represent each of the population characteristics, by the following criteria:

1. The company was included in Indonesia's manufacturing industry.

2. Manufacturing Company has published a complete financial report that available during period of study, 2013 and 2014.

3. The Manufacturing Company was not in a state of merger or liquidation.

Sampling used in this study was 125 manufacturing companies listed at Indonesia Stock Exchange (BEI), 14 of them were not used as the sample because of incomplete financial report, such as: WTON, ISSP, ETWA, SOBI, AKPI, SIPD, MYTX, IKBI, VOKS, AISA, ALTO, UNVR, CINT, KDSI.

\section{Research Variable}

This study used 2 variables, namely dependent variable and independent variable. A dependent variable was company's bankruptcy status while an independent variable was Liquidity (X1), Profitability in certain period (X2), Profitability (X3), Financial Structure (X4), and a Capital Turnover (X4):

\section{Research Model}

$Z$-Score calculation was started by calculating used variables then entered those variable score into $Z$-Score to obtain $Z$ score. The variable consisted of Liquidity Ratio (X1), Age of Firm and Cumulative Profitability Ratio (X2), Profitability Ratio (X3), Financial Structure Ratio (X4), and Capital Turnover Ratio (X5)

$Z$-Score can be calculated using:

$Z$-Score $=0,717 \mathrm{X} 1+0,847 \mathrm{X} 2+3,107 \mathrm{X} 3+0,420 \mathrm{X} 4+0,998 \mathrm{X} 5$

In which:

$\mathrm{X} 1=$ (Current Asset - Current Liabilities) $/$ Total Asset

$\mathrm{X} 2=$ Retained Earning / Total Asset

$\mathrm{X} 3=\mathrm{EBIT} /$ Total Asset

X4 = Market Value Equity / book value of Total Debt

$\mathrm{X} 5$ = Sales $/$ Total Asset

\section{Z-Score Indication}

$<1.81 \quad$ Bankrupt

$1.81-2.99$

$>2.99$ Gray Area / zone of ignorance

Not Bankrupt

\section{Data Collection}

This study used quantitative data. The data was secondary data, in the form of Publication Financial Statement Manufacturing Company data published at Indonesia Stock Exchange and website: www.idx.com.

The literature review was collected from any sources such as theses, journals, articles, newspapers, books, etc. The researcher collected, studied and noted the necessary data that gathered from various sources in STEI's library or other library. The technique used in collecting the data was literature research (Library research). As well as observation in directly observing research object to get the real situation of the object obtained. Through this field research (field research) the researcher wanted to know the consistency test, thus the researcher would obtain a relevant and accurate data. 


\section{Data Analysis Method}

The method of analysis used by researcher to process the data was using Altman $Z$-Score method and level of conformity test with the actual reality.

The stage that was used in this study to determine financial condition using Altman Z-Score were:

1. Calculating financial ratio

2. Conducting a calculation with discriminant analysis found by Altman through following formula:

$Z$-Score $=0,717 \mathrm{X} 1+0,847 \mathrm{X} 2+3,107 \mathrm{X} 3+0,420 \mathrm{X} 4+0,998 \mathrm{X} 5$

3. Conducting an interpretation of processed $Z$-Score result calculation. This was certainly with the limit that has been determined,

$$
Z \text {-Score Indication }
$$

$<1.81$ Bankrupt

$1.81-2.99$

$>2.99$ Gray Area / zone of ignorance

Not Bankrupt

4. Arranged a ranking index/ranking based on $z$-score.

In this study, the researcher used historical analysis to determine conformity level with actual reality.

\section{Data Collection}

This study used quantitative data. The data was secondary data, in the form of Publication Financial Statement Manufacturing Company data published at Indonesia Stock Exchange and website: www.idx.com.

The literature review was collected from any sources such as theses, journals, articles, newspapers, books, etc. The researcher collected, studied and noted the necessary data that gathered from various sources in STEI's library or other library.

The technique used in collecting the data was literature research (Library research). As well as observation in directly observing research object to get the real situation of the object obtained. Through this field research (field research) the researcher wanted to know the consistency test, thus the researcher would obtain a relevant and accurate data.

\section{Data Analysis Method}

The method of analysis used by researcher to process the data was using Altman $Z$-Score method and level of conformity test with the actual reality.

The stage that was used in this study to determine financial condition using Altman Z-Score were:

1. Calculating financial ratio

2. Conducting a calculation with discriminant analysis found by Altman through following formula:

$$
Z \text {-Score }=0,717 \mathrm{X} 1+0,847 \mathrm{X} 2+3,107 \mathrm{X} 3+0,420 \mathrm{X} 4+0,998 \mathrm{X} 5
$$

3. Conducting an interpretation of processed $Z$-Score result calculation. This was certainly with the limit that has been determined,

$$
\begin{array}{ll}
\frac{Z \text {-Score }}{<1.81} & \underline{\text { Indication }} \\
1.81-2.99 & \text { Graykrupt Area / zone of ignorance } \\
>2.99 & \text { Not Bankrupt }
\end{array}
$$

4. Arranged a ranking index/ranking based on $z$-score.

In this study, the researcher used historical analysis to determine conformity level with actual reality. 


\section{FINDINGS AND DISCUSSION}

\section{Altman Z-Score Model Analysis}

Altman score calculation is presented in Table 1 below.

Table 1.

Company Rating Index Result in 2013

\begin{tabular}{llc}
\hline \multicolumn{1}{c}{ Code } & Z-Score & Ranking \\
\hline INCI & 5.2488 & 1 \\
\hline MLBI & 5.2049 & 2 \\
\hline HMSP & 5.0123 & 3 \\
\hline ALKA & 4.7677 & 4 \\
\hline DLTA & 4.7268 & 5 \\
\hline JPRS & 4.0087 & 6 \\
\hline SIDO & 3.9675 & 7 \\
\hline KIAS & 3.8184 & 8 \\
\hline DPNS & 3.5497 & 9 \\
\hline
\end{tabular}

Source: Data analysis result

In Table 1, a company with INCI code is PT. Intan Wijaya Internasional Tbk in chemical subsector that has 5,2488 $z$-score. The company has an indication higher than 2,99 so that it is on healthy condition and the first rank of manufacturing company in 2013. Next, the company with SQBB code is PT. Taisho Pharmaceutical Indonesia Tbk in pharmacy subsector that has 3,0478 z-score by an indication is higher than 2,99. Consequently, the company is in health condition and ranked 19th in manufacturing company in 2013.

Table 2.

Company Rating Index Result in 2014

\begin{tabular}{llc}
\hline \multicolumn{1}{c}{ Code } & Z-Score & Ranking \\
\hline SIDO & 5.3240 & 1 \\
\hline ALKA & 5.3204 & 2 \\
\hline INCI & 5.1253 & 3 \\
\hline SIAP & 5.0890 & 4 \\
\hline HMSP & 4.5178 & 5 \\
\hline DLTA & 4.4395 & 6 \\
\hline KIAS & 4.2866 & 7 \\
\hline IGAR & 3.7644 & 8 \\
\hline JPRS & 3.7240 & 9 \\
\hline CEKA & 3.6981 & 10 \\
\hline SMBR & 3.5237 & 11 \\
\hline TBMS & 3.3968 & 12 \\
\hline KICI & 3.3446 & 13 \\
\hline KBLI & 3.3319 & 14 \\
\hline LMSH & 3.2977 & 15 \\
\hline SKBM & 3.2340 & 16 \\
\hline MERK & 3.2219 & 17 \\
\hline SMSM & 3.1994 & 18 \\
\hline TFCO & 3.1983 & 19 \\
\hline SCCO & 3.1818 & 20 \\
\hline SQBB & 3.1069 & 21 \\
\hline KLBF & 3.0989 & 22 \\
\hline
\end{tabular}

Source: Data analysis result 
In Table 2, a company with SIDO code is PT. Industri Jamu \& Farmasi Sido Muncul Tbk in pharmacy subsector that has 5,3240 z-score by an indication is upper than 2,99 . The result means that a company is in healthy position and ranked/highest rank in manufacturing company at Indonesia Stock Exchange(BEI) in 2014. Next, the company with KLBF code is PT. Kalbe Farma Tbk in pharmaceutical subsector that has $3,0989 \mathrm{z}$-score by an indication is upper than 2,99 which is ranked 22nd in manufacturing company at Indonesia Stock Exchange (BEI) in 2014.

Table 3.

2013 Gray Area indication ranking index

\begin{tabular}{|c|c|c|}
\hline Code & Z-Score & Ranking \\
\hline GDST & 2.9706 & 1 \\
\hline KBRI & 2.9001 & 2 \\
\hline KICI & 2.8959 & 3 \\
\hline KLBF & 2.8859 & 4 \\
\hline SMSM & 2.8738 & 5 \\
\hline $\mathrm{SCCO}$ & 2.8641 & 6 \\
\hline GDYR & 2.8344 & 7 \\
\hline KAEF & 2.8123 & 8 \\
\hline ARNA & 2.7721 & 9 \\
\hline TCID & 2.7630 & 10 \\
\hline MAIN & 2.6945 & 11 \\
\hline SRSN & 2.6764 & 12 \\
\hline ULTJ & 2.6647 & 13 \\
\hline PSDN & 2.6584 & 14 \\
\hline TSPC & 2.6468 & 15 \\
\hline INTP & 2.6431 & 16 \\
\hline DVLA & 2.6241 & 17 \\
\hline LION & 2.6035 & 18 \\
\hline TFCO & 2.5993 & 19 \\
\hline TRIS & 2.5941 & 20 \\
\hline UNIC & 2.5723 & 21 \\
\hline EKAD & 2.4611 & 22 \\
\hline WIIM & 2.4523 & 23 \\
\hline BTON & 2.3757 & 24 \\
\hline SKLT & 2.3597 & 25 \\
\hline TOTO & 2.2959 & 26 \\
\hline BATA & 2.2955 & 27 \\
\hline AMFG & 2.2934 & 28 \\
\hline MYOR & 2.2711 & 29 \\
\hline ADES & 2.1853 & 30 \\
\hline PBRX & 2.1592 & 31 \\
\hline CTBN & 2.1495 & 32 \\
\hline GGRM & 2.1481 & 33 \\
\hline SMGR & 2.1367 & 34 \\
\hline JPFA & 2.0351 & 35 \\
\hline ALDO & 1.9958 & 36 \\
\hline MRAT & 1.9838 & 27 \\
\hline FPNI & 1.9585 & 28 \\
\hline INDS & 1.9487 & 29 \\
\hline APLI & 1.9283 & 40 \\
\hline MBTO & 1.9242 & 41 \\
\hline ICBP & 1.9210 & 42 \\
\hline KBLM & 1.9079 & 43 \\
\hline STTP & 1.8777 & 44 \\
\hline SRIL & 1.8466 & 45 \\
\hline
\end{tabular}

Source: Data analysis result 
In Table 3, it is GDST code, that is PT. Gunawan Dianjaya Steel Tbk in metal subsector that has $2,9706 \mathrm{z}$-score by an indication of $1,81-2,99$. The result means that a company is in gray area and ranked the highest in manufacturing company at Indonesia Stock Exchange (BEI) in 2013. Further, PT Sri Rejeki Isman Tbk with SRIL code in textile $\&$ garment subsector that has $1,8466 \mathrm{z}$-score by an indication is 1,81 2 ,99. The results indicates that a company is ranked on 45th in manufacturing company in 2013.

Table 4.

2014 Gray Area indication ranking index

\begin{tabular}{|c|c|c|}
\hline Code & Z-Score & Ranking \\
\hline APLI & 2.8984 & 1 \\
\hline ARNA & 2.8981 & 2 \\
\hline ULTJ & 2.8666 & 3 \\
\hline MLBI & 2.7971 & 4 \\
\hline KAEF & 2.6743 & 5 \\
\hline PTSN & 2.6612 & 6 \\
\hline TSPC & 2.6492 & 7 \\
\hline SKLT & 2.6446 & 8 \\
\hline UNIC & 2.6239 & 9 \\
\hline DPNS & 2.5935 & 10 \\
\hline EKAD & 2.5865 & 11 \\
\hline FPNI & 2.5765 & 12 \\
\hline INTP & 2.5326 & 13 \\
\hline SRSN & 2.4899 & 14 \\
\hline AMFG & 2.4800 & 15 \\
\hline DVLA & 2.4378 & 16 \\
\hline BATA & 2.3253 & 17 \\
\hline TCID & 2.3199 & 18 \\
\hline TOTO & 2.3149 & 19 \\
\hline WIIM & 2.2927 & 20 \\
\hline TRIS & 2.2797 & 21 \\
\hline CPIN & 2.2734 & 22 \\
\hline GDYR & 2.2272 & 23 \\
\hline GGRM & 2.1585 & 24 \\
\hline SMGR & 2.1203 & 25 \\
\hline ICBP & 2.1166 & 26 \\
\hline MYOR & 2.0937 & 27 \\
\hline STTP & 2.0853 & 28 \\
\hline LION & 2.0637 & 29 \\
\hline INDS & 2.0280 & 30 \\
\hline BTON & 2.0263 & 31 \\
\hline JPFA & 2.0118 & 32 \\
\hline PSDN & 2.0095 & 33 \\
\hline IMPC & 2.0068 & 34 \\
\hline PYFA & 1.9750 & 35 \\
\hline ALDO & 1.9571 & 36 \\
\hline MBTO & 1.9396 & 37 \\
\hline MRAT & 1.9268 & 38 \\
\hline KBLM & 1.9117 & 39 \\
\hline ADES & 1.8977 & 40 \\
\hline CTBN & 1.8696 & 41 \\
\hline
\end{tabular}

Source: Data analysis result 
Table 4 shows that a company with APLI code is PT. Asiaplast Industries Tbk in plastic subsector $\&$ packaging that has $2,8984 \mathrm{z}$-score by an indication of $1,81-2,99$. Consequently, the company is in gray area and ranked the first in manufacturing company at Indonesia Stock Exchange (BEI) in 2014. Further, the company with CTBN code is PT. Citra Tubindo Tbk in metal subsector that has 1,8696 z-score by an indication of $1,81-2,99$. The company is in gray area, ranked 41 st in manufacturing company at Indonesia Stock Exchange (BEI) in 2014.

Table 5.

2013 Bankrupt Indication Ranking Index Result

\begin{tabular}{cccccc}
\hline Code & Z-Score & Ranking & Code & Z-Score & Ranking \\
\hline PYFA & 1.8020 & 1 & LPIN & 1.0313 & 32 \\
\hline BRAM & 1.7772 & 2 & SIAP & 1.0263 & 33 \\
\hline IMPC & 1.7567 & 3 & RMBA & 0.9974 & 34 \\
\hline TPIA & 1.7238 & 4 & YPAS & 0.9937 & 35 \\
\hline SMBR & 1.7212 & 5 & FASW & 0.9312 & 36 \\
\hline ASII & 1.6694 & 6 & BRNA & 0.9301 & 37 \\
\hline NIKL & 1.6478 & 7 & MASA & 0.9109 & 38 \\
\hline AUTO & 1.6289 & 8 & SSTM & 0.8759 & 39 \\
\hline ADMG & 1.6148 & 9 & TKIM & 0.8661 & 40 \\
\hline PICO & 1.5742 & 10 & BAJA & 0.8201 & 41 \\
\hline NIPS & 1.5197 & 11 & SCPI & 0.7763 & 42 \\
\hline ROTI & 1.4788 & 12 & LMPI & 0.7710 & 43 \\
\hline ERTX & 1.4296 & 13 & INKP & 0.7616 & 44 \\
\hline RICY & 1.3824 & 14 & JKSW & 0.5916 & 45 \\
\hline INDR & 1.3730 & 15 & PRAS & 0.5689 & 46 \\
\hline BUDI & 1.3716 & 16 & IKAI & 0.5324 & 47 \\
\hline JECC & 1.3686 & 17 & ESTI & 0.5159 & 48 \\
\hline BRPT & 1.3637 & 18 & CNTX & 0.5006 & 49 \\
\hline STAR & 1.3629 & 19 & UNIT & 0.2977 & 50 \\
\hline IPOL & 1.3297 & 20 & MLIA & 0.1453 & 51 \\
\hline GJTL & 1.3033 & 21 & TIRT & 0.0761 & 52 \\
\hline SMCB & 1.2687 & 22 & BIMA & 0.0064 & 53 \\
\hline ALMI & 1.2640 & 23 & HDTX & $(0,0516)$ & 54 \\
\hline DAJK & 1.2414 & 24 & SIMA & $(0,1671)$ & 55 \\
\hline INDF & 1.2123 & 25 & ARGO & $(0,1800)$ & 56 \\
\hline INAF & 1.1638 & 26 & AKKU & $(0,1932)$ & 57 \\
\hline KRAS & 1.1535 & 27 & INRU & $(0,4951)$ & 58 \\
\hline IMAS & 1.1149 & 28 & UNTX & $(0,9859)$ & 59 \\
\hline SPMA & 1.0937 & 29 & SULI & $(2,6519)$ & 60 \\
\hline TRST & 1.0856 & 30 & POLY & $(5,2665)$ & 61 \\
\hline INAI & 1.0510 & 31 & & & \\
\hline & & & & \\
\hline Sou & $D$ & & & & 51 \\
\hline
\end{tabular}

Source: Data analysis result

Table 5 shows that a company with PYFA is PT. Pyridam Farma Tbk in pharmacy sub sector that has $1,8020 \mathrm{z}$-score by an indication of $<1,81$. The result means that a company is in bankrupt position and ranked/ highest ranked in manufacturing company at Indonesia Stock Exchange (BEI) in 2013. Further, the company with POLY code is PT. Asia Pacific Fibers Tbk in textile $\&$ garment sub sector that has $(5,2665) z$-score by an indication of $<1,81$. The company is ranked in 61 st in manufacturing company in 2013. 
Table 6.

2014 Bankrupt Indication Ranking Index Result

\begin{tabular}{|c|c|c|c|c|c|}
\hline Code & Z-Score & Ranking & Code & Z-Score & Ranking \\
\hline TPIA & 1.7036 & 1 & STAR & 1.2120 & 32 \\
\hline RICY & 1.6999 & 2 & TIRT & 1.2115 & 33 \\
\hline PICO & 1.6863 & 3 & BUDI & 1.2099 & 34 \\
\hline GDST & 1.661 & 4 & ALMI & 1.1612 & 35 \\
\hline SRIL & 1.6290 & 5 & LMPI & 1.1308 & 36 \\
\hline JECC & 1.6248 & 6 & KRAS & 1.0439 & 37 \\
\hline ASII & 1.5970 & 7 & SMCB & 1.0374 & 38 \\
\hline ROTI & 1.5856 & 8 & IMAS & 0.9259 & 39 \\
\hline PBRX & 1.5735 & 9 & MASA & 0.9066 & 40 \\
\hline YPAS & 1.5703 & 10 & MLIA & 0.8954 & 41 \\
\hline AUTO & 1.4785 & 11 & SCPI & 0.8349 & 42 \\
\hline ERTX & 1.4645 & 12 & TKIM & 0.8175 & 43 \\
\hline ADMG & 1.4601 & 13 & SSTM & 0.7918 & 44 \\
\hline INDF & 1.4472 & 14 & INKP & 0.7650 & 45 \\
\hline BRAM & 1.4353 & 15 & LPIN & 0.7104 & 46 \\
\hline INAF & 1.4261 & 16 & RMBA & 0.6905 & 47 \\
\hline MAIN & 1.4044 & 17 & CNTX & 0.6671 & 48 \\
\hline BRPT & 1.3979 & 18 & SIMA & 0.5914 & 49 \\
\hline INDR & 1.3650 & 19 & JKSW & 0.4602 & 50 \\
\hline TRST & 1.3597 & 20 & PRAS & 0.4575 & 51 \\
\hline NIKL & 1.3282 & 21 & UNIT & 0.3641 & 52 \\
\hline GJTL & 1.3072 & 22 & ESTI & 0.2116 & 53 \\
\hline DAJK & 1.3046 & 23 & IKAI & 0.1182 & 54 \\
\hline BRNA & 1.3016 & 24 & AKKU & $(0,1055)$ & 55 \\
\hline NIPS & 1.2905 & 25 & HDTX & $(0,1851)$ & 56 \\
\hline SPMA & 1.2840 & 26 & INRU & $(0,3987)$ & 57 \\
\hline BAJA & 1.2765 & 27 & KBRI & $(0,7327)$ & 58 \\
\hline INAI & 1.2710 & 28 & UNTX & $(0,9026)$ & 59 \\
\hline IPOL & 1.2465 & 29 & SULI & $(0,9179)$ & 60 \\
\hline BIMA & 1.2357 & 30 & ARGO & $(1,3555)$ & 61 \\
\hline FASW & 1.2333 & 31 & POLY & $(8,0419)$ & 62 \\
\hline
\end{tabular}

Source: Data analysis result

In Table 6 a company with TPIA code is PT. Chandra Asri Petrochemical Tbk in pharmacy sub sector that has $1,7036 \mathrm{z}$-score by an indication of $<1,81$. Consequently, the company is in bankrupt and ranked/first ranking in manufacturing company in 2014. Further, the company with POLI code is PT. Asia Pacific Fiber Tbk in textille \& garment that has $(8,0419) z$-score by an indication $<1,81$. Consequently, the company is in bankrupt and ranked/ranking 62 nd or the lowest in manufacturing company in 2014.

Table 7.

Company Health Level Percentage

\begin{tabular}{lcc}
\hline Indication & 2013 & 2014 \\
\hline Healthy & $15,2 \%$ & $17,6 \%$ \\
\hline Gray area & $36 \%$ & $32,8 \%$ \\
\hline Bankrupt & $48,8 \%$ & $49,6 \%$ \\
\hline
\end{tabular}

Source: Data analysis result 
Based on the researcher study on bankruptcy indication uses Altman Z-Score method in Table 4.7. indicates that in 2014 at Indonesia Stock Exchange (BEI) there are 125 manufacturing companies from various sector with the percentage of $49,6 \%$ is in bankrupt, $32,8 \%$ is in gray area, and $17,6 \%$ in healthy position. The healthy position is fewer than other, this means to be not good in manufacturing company at Indonesia Stock Exchange (BEI) in 2013 at Indonesia Stock Exchange there are 125 manufacturing companies from various sector with the percentage of percentage of $48,8 \%$ is in bankrupt and $36 \%$ is in gray area and $15,2 \%$ is in healthy position. The healthy position is fewer than the other; this means to be not good in manufacturing company at Indonesia Stock Exchange (BEI).

\section{Conformity with Actual Reality Analysis}

Table 8

Conformity with Actual Reality Tahun 2013 - First I

\begin{tabular}{|c|c|c|c|c|c|c|c|}
\hline No & Code & $\begin{array}{l}2013 \text { Z- } \\
\text { Score }\end{array}$ & $\begin{array}{l}2014 \\
\text { bankrupt / no } \\
\text { bankrupt }\end{array}$ & No & Code & $\begin{array}{l}2013 Z- \\
\text { Score }\end{array}$ & $\begin{array}{l}2014 \\
\text { bankrupt / no } \\
\text { bankrupt }\end{array}$ \\
\hline 1 & INCI & Healthy & not bankrupt & 37 & LION & gray area & not bankrupt \\
\hline 2 & MLBI & Healthy & not bankrupt & 38 & TFCO & gray area & not bankrupt \\
\hline 3 & HMSP & Healthy & not bankrupt & 39 & TRIS & gray area & not bankrupt \\
\hline 4 & ALKA & Healthy & not bankrupt & 40 & UNIC & gray area & not bankrupt \\
\hline 5 & DLTA & Healthy & not bankrupt & 41 & EKAD & gray area & not bankrupt \\
\hline 6 & JPRS & Healthy & not bankrupt & 42 & WIIM & gray area & not bankrupt \\
\hline 7 & SIDO & Healthy & not bankrupt & 43 & BTON & gray area & not bankrupt \\
\hline 8 & KIAS & Healthy & not bankrupt & 44 & SKLT & gray area & not bankrupt \\
\hline 9 & DPNS & Healthy & not bankrupt & 45 & TOTO & gray area & not bankrupt \\
\hline 10 & TBMS & Healthy & not bankrupt & 46 & BATA & gray area & not bankrupt \\
\hline 11 & SKBM & Healthy & not bankrupt & 47 & AMFG & gray area & not bankrupt \\
\hline 12 & IGAR & Healthy & not bankrupt & 48 & MYOR & gray area & not bankrupt \\
\hline 13 & LMSH & Healthy & not bankrupt & 49 & ADES & gray area & not bankrupt \\
\hline 14 & PTSN & Healthy & not bankrupt & 50 & PBRX & gray area & not bankrupt \\
\hline 15 & CEKA & Healthy & not bankrupt & 51 & CTBN & gray area & not bankrupt \\
\hline 16 & CPIN & Healthy & not bankrupt & 52 & GGRM & gray area & not bankrupt \\
\hline 17 & MERK & Healthy & not bankrupt & 53 & SMGR & gray area & not bankrupt \\
\hline 18 & KBLI & Healthy & not bankrupt & 54 & JPFA & gray area & not bankrupt \\
\hline 19 & SQBB & Healthy & not bankrupt & 55 & ALDO & gray area & not bankrupt \\
\hline 20 & GDST & gray area & not bankrupt & 56 & MRAT & gray area & not bankrupt \\
\hline 21 & KBRI & gray area & not bankrupt & 57 & FPNI & gray area & not bankrupt \\
\hline 22 & KICI & gray area & not bankrupt & 58 & INDS & gray area & not bankrupt \\
\hline 23 & KLBF & gray area & not bankrupt & 59 & APLI & gray area & not bankrupt \\
\hline 24 & SMSM & gray area & not bankrupt & 60 & MBTO & gray area & not bankrupt \\
\hline 25 & $\mathrm{SCCO}$ & gray area & not bankrupt & 61 & ICBP & gray area & not bankrupt \\
\hline 26 & GDYR & gray area & not bankrupt & 62 & KBLM & gray area & not bankrupt \\
\hline 27 & KAEF & gray area & not bankrupt & 63 & STTP & gray area & not bankrupt \\
\hline 28 & ARNA & gray area & not bankrupt & 64 & SRIL & gray area & not bankrupt \\
\hline 29 & TCID & gray area & not bankrupt & 65 & PYFA & bankrupt & not bankrupt \\
\hline 30 & MAIN & gray area & not bankrupt & 66 & BRAM & bankrupt & not bankrupt \\
\hline 31 & SRSN & gray area & not bankrupt & 67 & IMPC & bankrupt & not bankrupt \\
\hline 32 & ULTJ & gray area & not bankrupt & 68 & TPIA & bankrupt & not bankrupt \\
\hline 33 & PSDN & gray area & not bankrupt & 69 & SMBR & bankrupt & not bankrupt \\
\hline 34 & TSPC & gray area & not bankrupt & 70 & ASII & bankrupt & not bankrupt \\
\hline 35 & INTP & gray area & not bankrupt & 71 & NIKL & bankrupt & not bankrupt \\
\hline 36 & DVLA & gray area & not bankrupt & 72 & AUTO & bankrupt & not bankrupt \\
\hline
\end{tabular}


Table 9

Conformity with Actual Reality 2013 - Part II

\begin{tabular}{|c|c|c|c|c|c|c|c|}
\hline No & Code & $\begin{array}{l}2013 \text { Z- } \\
\text { Score }\end{array}$ & $\begin{array}{l}2014 \\
\text { bankrupt / no } \\
\text { bankrupt }\end{array}$ & No & Code & $\begin{array}{l}2013 \text { Z- } \\
\text { Score }\end{array}$ & $\begin{array}{l}2014 \\
\text { bankrupt / no } \\
\text { bankrupt }\end{array}$ \\
\hline 73 & ADMG & bankrupt & not bankrupt & 99 & YPAS & bankrupt & not bankrupt \\
\hline 74 & $\mathrm{PICO}$ & bankrupt & not bankrupt & 100 & FASW & bankrupt & not bankrupt \\
\hline 75 & NIPS & bankrupt & not bankrupt & 101 & BRNA & bankrupt & not bankrupt \\
\hline 76 & ROTI & bankrupt & not bankrupt & 102 & MASA & bankrupt & not bankrupt \\
\hline 77 & ERTX & bankrupt & not bankrupt & 103 & SSTM & bankrupt & not bankrupt \\
\hline 78 & RICY & bankrupt & not bankrupt & 104 & TKIM & bankrupt & not bankrupt \\
\hline 79 & INDR & bankrupt & not bankrupt & 105 & BAJA & bankrupt & not bankrupt \\
\hline 80 & BUDI & bankrupt & not bankrupt & 106 & SCPI & bankrupt & not bankrupt \\
\hline 81 & JECC & bankrupt & not bankrupt & 107 & LMPI & bankrupt & not bankrupt \\
\hline 82 & BRPT & bankrupt & not bankrupt & 108 & INKP & bankrupt & not bankrupt \\
\hline 83 & STAR & bankrupt & not bankrupt & 109 & JKSW & bankrupt & not bankrupt \\
\hline 84 & IPOL & bankrupt & not bankrupt & 110 & PRAS & bankrupt & not bankrupt \\
\hline 85 & GJTL & bankrupt & not bankrupt & 111 & IKAI & bankrupt & not bankrupt \\
\hline 86 & SMCB & bankrupt & not bankrupt & 112 & ESTI & bankrupt & not bankrupt \\
\hline 87 & ALMI & bankrupt & not bankrupt & 113 & CNTX & bankrupt & not bankrupt \\
\hline 88 & DAJK & bankrupt & not bankrupt & 114 & UNIT & bankrupt & not bankrupt \\
\hline 89 & INDF & bankrupt & not bankrupt & 115 & MLIA & bankrupt & not bankrupt \\
\hline 90 & INAF & bankrupt & not bankrupt & 116 & TIRT & bankrupt & not bankrupt \\
\hline 91 & KRAS & bankrupt & not bankrupt & 117 & BIMA & bankrupt & not bankrupt \\
\hline 92 & IMAS & bankrupt & not bankrupt & 118 & HDTX & bankrupt & not bankrupt \\
\hline 93 & SPMA & bankrupt & not bankrupt & 119 & SIMA & bankrupt & not bankrupt \\
\hline 94 & TRST & bankrupt & not bankrupt & 120 & ARGO & bankrupt & not bankrupt \\
\hline 95 & INAI & bankrupt & not bankrupt & 121 & AKKU & bankrupt & not bankrupt \\
\hline 96 & LPIN & bankrupt & not bankrupt & 122 & INRU & bankrupt & not bankrupt \\
\hline 97 & SIAP & bankrupt & not bankrupt & 123 & UNTX & bankrupt & not bankrupt \\
\hline 98 & RMBA & bankrupt & not bankrupt & 124 & SULI & bankrupt & not bankrupt \\
\hline
\end{tabular}

Source: Data analysis result

Table 10

Conformity with Actual Reality Tahun 2014 - Part I

\begin{tabular}{|r|l|l|l|r|l|l|l|}
\hline No & Code & $\begin{array}{l}2014 \text { Z- } \\
\text { Score }\end{array}$ & $\begin{array}{l}2015 \\
\text { bankrupt / no } \\
\text { bankrupt }\end{array}$ & No & Code & $\begin{array}{l}2014 \text { Z- } \\
\text { Score }\end{array}$ & $\begin{array}{l}2015 \\
\text { bankrupt / no } \\
\text { bankrupt }\end{array}$ \\
\hline 1 & SIDO & Healthy & not bankrupt & 19 & TFCO & Healthy & not bankrupt \\
\hline 2 & ALKA & Healthy & not bankrupt & 20 & SCCO & Healthy & not bankrupt \\
\hline 3 & INCI & Healthy & not bankrupt & 21 & SQBB & Healthy & not bankrupt \\
\hline 4 & SIAP & Healthy & not bankrupt & 22 & KLBF & Healthy & not bankrupt \\
\hline 5 & HMSP & Healthy & not bankrupt & 23 & APLI & gray area & not bankrupt \\
\hline 6 & DLTA & Healthy & not bankrupt & 24 & ARNA & gray area & not bankrupt \\
\hline 7 & KIAS & Healthy & not bankrupt & 25 & ULTJ & gray area & not bankrupt \\
\hline 8 & IGAR & Healthy & not bankrupt & 26 & MLBI & gray area & not bankrupt \\
\hline 9 & JPRS & Healthy & not bankrupt & 27 & KAEF & gray area & not bankrupt \\
\hline 10 & CEKA & Healthy & not bankrupt & 28 & PTSN & gray area & not bankrupt \\
\hline 11 & SMBR & Healthy & not bankrupt & 29 & TSPC & gray area & not bankrupt \\
\hline 12 & TBMS & Healthy & not bankrupt & 30 & SKLT & gray area & not bankrupt \\
\hline 13 & KICI & Healthy & not bankrupt & 31 & UNIC & gray area & not bankrupt \\
\hline 14 & KBLI & Healthy & not bankrupt & 32 & DPNS & gray area & not bankrupt \\
\hline 15 & LMSH & Healthy & not bankrupt & 33 & EKAD & gray area & not bankrupt \\
\hline 16 & SKBM & Healthy & not bankrupt & 34 & FPNI & gray area & not bankrupt \\
\hline 17 & MERK & Healthy & not bankrupt & 35 & INTP & gray area & not bankrupt \\
\hline 18 & SMSM & Healthy & not bankrupt & 36 & SRSN & gray area & not bankrupt \\
\hline
\end{tabular}


Table 11

Conformity with Actual Reality 2014 - Part II

\begin{tabular}{|c|c|c|c|c|c|c|c|}
\hline No & Code & $\begin{array}{l}2014 Z- \\
\text { Score }\end{array}$ & $\begin{array}{l}2015 \\
\text { bankrupt / no } \\
\text { bankrupt }\end{array}$ & No & Code & $\begin{array}{l}2014 Z- \\
\text { Score }\end{array}$ & $\begin{array}{l}2015 \\
\text { bankrupt / no } \\
\text { bankrupt }\end{array}$ \\
\hline 37 & AMFG & gray area & not bankrupt & 83 & TRST & bankrupt & not bankrupt \\
\hline 38 & DVLA & gray area & not bankrupt & 84 & NIKL & bankrupt & not bankrupt \\
\hline 39 & BATA & gray area & not bankrupt & 85 & GJTL & bankrupt & not bankrupt \\
\hline 40 & TCID & gray area & not bankrupt & 86 & DAJK & bankrupt & not bankrupt \\
\hline 41 & TOTO & gray area & not bankrupt & 87 & BRNA & bankrupt & not bankrupt \\
\hline 42 & WIIM & gray area & not bankrupt & 88 & NIPS & bankrupt & not bankrupt \\
\hline 43 & TRIS & gray area & not bankrupt & 89 & SPMA & bankrupt & not bankrupt \\
\hline 44 & CPIN & gray area & not bankrupt & 90 & BAJA & bankrupt & not bankrupt \\
\hline 45 & GDYR & gray area & not bankrupt & 91 & INAI & bankrupt & not bankrupt \\
\hline 46 & GGRM & gray area & not bankrupt & 92 & IPOL & bankrupt & not bankrupt \\
\hline 47 & SMGR & gray area & not bankrupt & 93 & BIMA & bankrupt & not bankrupt \\
\hline 48 & ICBP & gray area & not bankrupt & 94 & FASW & bankrupt & not bankrupt \\
\hline 49 & MYOR & gray area & not bankrupt & 95 & STAR & bankrupt & not bankrupt \\
\hline 50 & STTP & gray area & not bankrupt & 96 & TIRT & bankrupt & not bankrupt \\
\hline 51 & LION & gray area & not bankrupt & 97 & BUDI & bankrupt & not bankrupt \\
\hline 52 & INDS & gray area & not bankrupt & 98 & ALMI & bankrupt & not bankrupt \\
\hline 53 & BTON & gray area & not bankrupt & 99 & LMPI & bankrupt & not bankrupt \\
\hline 54 & JPFA & gray area & not bankrupt & 100 & KRAS & bankrupt & not bankrupt \\
\hline 55 & PSDN & gray area & not bankrupt & 101 & SMCB & bankrupt & not bankrupt \\
\hline 56 & IMPC & gray area & not bankrupt & 102 & IMAS & bankrupt & not bankrupt \\
\hline 57 & PYFA & gray area & not bankrupt & 103 & MASA & bankrupt & not bankrupt \\
\hline 58 & ALDO & gray area & not bankrupt & 104 & MLIA & bankrupt & not bankrupt \\
\hline 59 & MBTO & gray area & not bankrupt & 105 & SCPI & bankrupt & not bankrupt \\
\hline 60 & MRAT & gray area & not bankrupt & 106 & TKIM & bankrupt & not bankrupt \\
\hline 61 & KBLM & gray area & not bankrupt & 107 & SSTM & bankrupt & not bankrupt \\
\hline 62 & ADES & gray area & not bankrupt & 108 & INKP & bankrupt & not bankrupt \\
\hline 63 & CTBN & gray area & not bankrupt & 109 & LPIN & bankrupt & not bankrupt \\
\hline 64 & TPIA & bankrupt & not bankrupt & 110 & RMBA & bankrupt & not bankrupt \\
\hline 65 & RICY & bankrupt & not bankrupt & 111 & CNTX & bankrupt & not bankrupt \\
\hline 66 & $\mathrm{PICO}$ & bankrupt & not bankrupt & 112 & SIMA & bankrupt & not bankrupt \\
\hline 67 & GDST & bankrupt & not bankrupt & 113 & JKSW & bankrupt & not bankrupt \\
\hline 68 & SRIL & bankrupt & not bankrupt & 114 & PRAS & bankrupt & not bankrupt \\
\hline 69 & JECC & bankrupt & not bankrupt & 115 & UNIT & bankrupt & not bankrupt \\
\hline 70 & ASII & bankrupt & not bankrupt & 116 & ESTI & bankrupt & not bankrupt \\
\hline 71 & ROTI & bankrupt & not bankrupt & 117 & IKAI & bankrupt & not bankrupt \\
\hline 72 & PBRX & bankrupt & not bankrupt & 118 & AKKU & bankrupt & not bankrupt \\
\hline 73 & YPAS & bankrupt & not bankrupt & 119 & HDTX & bankrupt & not bankrupt \\
\hline 74 & AUTO & bankrupt & not bankrupt & 120 & INRU & bankrupt & not bankrupt \\
\hline 75 & ERTX & bankrupt & not bankrupt & 121 & KBRI & bankrupt & not bankrupt \\
\hline 76 & ADMG & bankrupt & not bankrupt & 122 & UNTX & bankrupt & not bankrupt \\
\hline 77 & INDF & bankrupt & not bankrupt & 123 & SULI & bankrupt & not bankrupt \\
\hline 78 & BRAM & bankrupt & not bankrupt & 124 & ARGO & bankrupt & not bankrupt \\
\hline 79 & INAF & bankrupt & not bankrupt & 125 & POLY & bankrupt & not bankrupt \\
\hline 80 & MAIN & bankrupt & not bankrupt & & & & \\
\hline 81 & BRPT & bankrupt & not bankrupt & & & & \\
\hline 82 & INDR & bankrupt & not bankrupt & & & & \\
\hline
\end{tabular}

Source: Data analysis result 
In Table 8 to Table 11 , it is showed that company's operation is still running normally in the further year and a company is said running in good condition after observed by the researcher. The results show that there is no conformity between index result and actual reality. Therefore, Altman z-score model index result cannot be used to predict company's bankruptcy, particularly in manufacturing industry listed in Indonesia stock exchange (IDX).

\section{CONCLUSION}

\section{Conclusion}

1. Manufacturing company's Z-Score index result in 2013 was occupied by PT. Intan Wijaya Internasional Tbk in pharmacy sub sector that ranked in the first place with a healthy condition, meanwhile the latest and the lowest rank was occupied by PT. Asia Pacific Fiber Tbk in textile \& garment with bankrupt.

2. Manufacturing company's Z-Score index result in 2014 was occupied by PT. Industri Jamu \& Farmasi Sido Muncul Tbk in pharmacy sub sector as the first rank with healthy condition, while the latest is PT. Asia Pacific Fiber Tbk in textile \& garment with bankrupt condition.

3. This result of the study is not consistent/suitable with the actual reality showing that Altman method cannot be used as company's bankruptcy trend tool.

\section{Limitation}

In this study there is limitation that can be taken into consideration for the next researcher in order to get better result. The limitations are:

a. Altman $z$-score analysis result is closely related to the limited number of sample and used data and period. So, this research is not able to analyze a whole part because Altman $z$-score analysis is limited to manufacturing company only so that it only describes manufacturing company's condition only.

b. Observation period that is only one year, it may give different result if it is conducted in longer period.

c. The used model in this study is only Altman Z-Score, basically there are several models to analyze bankruptcy, it may be different if it is used other model or several models.

\section{Suggestion}

The suggestions after conducting the study are:

1. Suggestion to manufacturing company

It is better that manufacturing company should always maintain and improve the company's performance even better, since based in 2013 and 2014 result, most of the company sampled have the potential to experience bankruptcy's indication with Z-Score value lower than 1.81 .

For manufacturing company, an Altman z-score model cannot be used to indicate a bankruptcy due to inconsistency with reality, but this model can help to assess and provide input for company's improvement and retention.

\section{Suggestion to further study:}

The researcher realizes that this research is not perfect at all. The researcher provides suggestions for further research. The number of sample and research's period are extended to produce better information. It is expected to be able to analyze other uncontrolled variable in this study or use various models for bankruptcy analysis such as Springate, Fulmer, Blasztk, etc. 


\section{REFERENCES}

Asy'ari, M Asim; Prasetyono and Haryadi, Bambang. 2014. Memetakan Satuan Pengawasan Intern Pada Pt X Dalam Kerangka Four Stage Model. Journal of Auditing, Financeand Forensic Accounting. Vol. 2 No. 1 April 2014. Hal 23-8

Bastian, Indra. 2009. Akuntansi Sektor Publik di Indonesia. Yogyakarta. BPFE Universitas Gajah Mada.

Desmiyawati dan Witaliza, Wulan. 2012. Pengaruh Komitmen organisasi, Pengendalian Intern, dan Akuntabilitas Publik Terhadap Kinerja Organisasi (Studi Empiris pada Rumah Sakit Swasta di Propinsi Riau). Jurnal Pekbis. Vol. 4. No. 1. hal 26-33.

Dewi, Sarita Permata. 2012. Pengaruh Pengendalian Internal dan Gaya Kepemimpinan terhadap Kinerja Karyawan SPBU Yogyakarta (Studi Kasus Pada SPBU Anak Cabang Perusahaan RB. Group). Jurnal Nominal. Vol. I, No.I.

Djajadikerta, Hamfri. 2004. Perbandingan Pengendalian Intern dan Pengendalian Manajemen dalam Hubungannya dengan Agency Theory, Jurnal Bina Ekonomi. Vol. 8. No.1. Hal. 1-109.

Ghany, Nur Farhaty. 2007. Pengaruh Budaya Organisasi Terhadap Kinerja Karyawan. Yogyakarta. Genta Press.

Ghozali, Imam. 2013. Aplikasi Analisis Multivariate dengan Program SPSS Edisi 7. Semarang. Badan Penerbit Universitas Diponegoro.

Halim, Abdul. 2007. Akuntansi Keuangan Daerah. Jakarta. Salemba Empat.

Ivancevich, John M. Robert Konopaske, and Michael T. Matteson. 2007. Perilaku dan Manajemen Organisasi. Edisi Ketujuh. Jakarta. Erlangga

Jensen, and W. H. Meckling, 1976, Theory of The Firm: Managerial Behavior, Agency Costs and Ownership Structure. Journal Of Financial Economics. Vol. 3. Pg. 305360.

Kusuma, I Gede Eka Arya. 2013. Pengaruh Kejelasan Sasaran Anggaran, Komitmen Organisasi dan Ketidakpastian Lingkungan pada Ketepatan Anggaran (Studi Empiris di SKPD Pemerintah Provinsi Bali). Tesis. Universitas Udayana

Kistoyo. 2008. Pengaruh Kepemimpinan, Komunikasi dan Lingkungan Fisik Terhadap Kinerja Pegawai Pada Kantor Dinas Pendidikan Kabupaten Pekalongan. Tesis, Universitas Sebelas Maret Surakarta.

Labni. 2015. Pengaruh Komitmen Organisasional, Sistem Pengendalian Intern Pemerintah, dan Akuntabilitas Publik Terhadap Kinerja Manajerial, Tesis. Universitas Mataram.

Locke, E.A., dan Latham, 1990, A Theory of Goal Setting and Task Performance, New Jersey, Prentice-Hall Inc.

Mardiasmo. 2009. Akuntansi Sektor Publik. Yogyakarta. Andi.

Mahsun, Mohammad. 2014. Pengukuran Kinerja Sektor Publik Edisi Pertama. Cetakan kelima. Yogyakarta. BPFE Universitas Gajah Mada.

Mahmudi. 2013. Manajemen Kinerja Sektor Publik Edisi Kedua Cetakan Kedua, Yogyakarta. UPP STIM.

Mulyadi, 2013, Sistem Akuntansi. Edisi 3, Cetakan 3, Jakarta, Salemba Empat

Mowday, Richard T, Steers, Richard M, Porter, Lyman W, 1979, The Measurement of Organization Commitmen. Journal of Vacational Behavior 14: 224-247.

Nawawi, H Hadari, 2006, Kepemimpinan Mengefektifkan Organisasi, Yogyakarta, Gadjah Mada University Press

Nasir, Azwir dan Oktari, Ranti, 2011, Pengaruh Pemanfaatan Teknologi Informasi, dan Pengendalian Intern Terhadap Kinerja Instansi Pemerintah (Studi pada Satuan Kerja Perangkat Daerah Kabupaten Kampar). Universitas Riau. 
Peraturan Pemerintah Nomor 58 Tahun 2005 tentang Pengelolaan Keuangan Daerah

Peraturan Pemerintah Nomor 60 Tahun 2008 tentang Sistem Pengendalian Intern Pemerintah.

Permendagri Nomor 13 Tahun 2006 tentang Pedoman Pengelolaan Keuangan Daerah.

Permen PAN dan RB Nomor 53Tahun 2014 tentang Petunjuk Teknis Perjanjian Kinerja, Pelaporan Kinerja dan Tata Cara Review atas Laporan Kinerja Intansi Pemerintah.

Pamungkas, Sum Rahmani. 2014. Pengaruh Gaya Kepemimpinan Terhadap Keefektifan Anggaran Partisipatif Dalam Peningkatan Kinerja Manajerial (Studi Kasus Pada Rumah Sakit Umum Daerah Di Wilayah Eks Karesidenan Surakarta). Universitas Muhammadiyah Surakarta.

Putri, Gustika Yolanda. 2013. Pengaruh Komitmen organisasi dan Sistem Pengendalian Intern Pemerintah (SPIP) Terhadap Kinerja manajerial SKPD (Studi Empiris pada Satuan Kerja Perangkat Daerah Kota Padang). Universitas Negeri Padang.

Ramandei, P. 2009. Pengaruh Karakteristik Sasaran Anggaran dan Sistem Pengendalian Intern Terhadap Kinerja Manajerial. Tesis. Universitas Diponegoro.

Rivai, Veithzal dan Mulyadi, Deddy. 2014. Kepemimpinan dan Perilaku Organisasi Edisi Ketiga. Jakarta. Raja Grafindo Persada.

Robbins, Stephen P. 2006. Perilaku Organisasi Edisi kesepuluh. Jakarta. Indeks Kelompok Gramedia.

Robbins, Stephen P, Judge, Timothy A. 2008. Perilaku Organisasi Edisi 12, Jakarta. Salemba Empat.

Sedarmayanti. 2004. Good Governance (Kepemerintahan yang Baik). Bandung. Mandar Maju.

Siregar, Sofian. 2013. Metode Penelitian Kuantitatif Dilengkapi dengan Perbandingan Perhitungan Manual \& SPSS. Jakarta. Kencana Prenadamedia Group.

Sugiyono. 2014. Metode Penelitian Bisnis, Cetakan Kedelapan Belas, Bandung, Alfa Beta.

Sudiarianti, Ni Made. 2015. Pengaruh Kompetensi Sumber Daya Manusia Pada Penerapan Sistem Pengendalian Intern Pemerintah dan Standar Akuntansi Pemerintah Serta Implikasinya Pada Kualitas Laporan Keuangan Daerah, Tesis, Universitas Udayana, Denpasar.

Supriyatno. 2010. Pengaruh Partisipasi Pejabat Struktural dalam Penyusunan Anggaran, Komitmen Organisasi, Profesionalisme dan Struktur Organisasi Pada Kinerja Manajerial Pemerintah Kota Denpasar. Tesis Universitas Udayana.

Sujihartini. 2013. Pengaruh Kepemimpinan, Motivasi dan Lingkungan Kerja Terhadap Kinerja Pegawai Pada Dinas Koperasi, Usaha Mikro Kecil dan Menengah Provinsi Nusa Tenggara Barat. Tesis. Universitas Mataram.

Sumarno, J. 2005. Pengaruh Komitmen Organisasi dan Gaya Kepemimpinan Terhadap Hubungan Antara Partisipasi Anggaran dan Kinerja Manajerial. Simposium Nasional Akuntansi VIII, Universitas Hasanuddin. Makasar.

Sumarno. 2006. Pengaruh Sistem Pengendalian Terhadap Kinerja Manajerial (Studi Kasus Pada Perusahaan Pelayaran Di Semarang). Tesis. Universitas Diponegoro, Semarang.

Tunti, Maria Elerina Douk. 2008. Analisa Pengaruh Kepuasan Kerja, Komitmen Organisasi dan Motivasi Terhadap Kinerja Manajeria Pemerintah Daerah. Tesis. Universitas Gadjah Mada.

Thoha, Miftah. 2013. Kepemimpinan Dalam Manajemen. Jakarta. Rajawali Pers.

Umam, Khaerul. 2010. Perilaku Organisasi. Bandung. Pustaka Setia.

Warisno. 2009. Faktor-Faktor yang Mempengaruhi Kinerja Satuan Kerja Perangkat Daerah (SKPD) di Lingkungan Pemerintah Provinsi Jambi. Tesis. Universitas SumateraUtara. 
